\title{
The Influence of KrF Excimer Laser Irradiation on the Surface of Collagen and Collagen/PVP Films
}

\author{
M. Wisniewski, ${ }^{1}$ A. Sionkowska, ${ }^{1}$ H. Kaczmarek, ${ }^{1}$ J. Skopinska, ${ }^{1}$ S. Lazare, ${ }^{2}$ and V. Tokarev $^{2}$ \\ ${ }^{1}$ Faculty of Chemistry, Nicolaus Copernicus University, Gagarin 7, 87-100 Toruń, Poland \\ ${ }^{2}$ Laboratoire de Physicochimie Moléculaire (LPCM), UMR 5803 du CNRS, Université de Bordeaux 1, \\ 351 Cours de la Libération, 33405 Talence, France
}

Received 19 February 2006; Revised 16 June 2006; Accepted 18 June 2006

\begin{abstract}
The surface properties of collagen and collagen/poly(vinyl pyrrolidone) (PVP) films after KrF excimer laser irradiation $(\lambda=$ $248 \mathrm{~nm}$ ) were investigated by means of the technique of scanning electron microscopy (SEM) and optical microscopy (OM). The laser irradiation of the used specimens caused an expansion of materials above their surface with pronounced sings of its melting. The height of the ablated volume in a case of collagen film at a fluence of $1.1 \mathrm{~J} / \mathrm{cm}^{2}$ was approximately $5 \mu \mathrm{m}$. A white damage appears on the treated surfaces at a threshold of $0.5 \mathrm{~J} / \mathrm{cm}^{2}$ with a single pulse and becomes more visible for a higher fluence. It is also noteworthy that along with increasing fluences (beginning from the fluence of $1.7 \mathrm{~J} / \mathrm{cm}^{2}$ ), the characteristic filaments at the spot edges are observed. The local heat and pressure generated by the laser radiation were able to induce bubble formation on the surface. Further bubble colliding and bursting produce a three-dimensional polymer "microfoam" structure with interconnected pores. These results can be of interest for some new applications.
\end{abstract}

Copyright (c) 2006 M. Wisniewski et al. This is an open access article distributed under the Creative Commons Attribution License, which permits unrestricted use, distribution, and reproduction in any medium, provided the original work is properly cited.

\section{INTRODUCTION}

The excimer laser-biomaterial interaction has recently attracted a great deal of attention in various scientific fields. For example, from the medical point of view, interests in biomaterials refer to the surgery methods using excimer lasers [1-6]. The biological components possess several properties relevant to pulsed laser ablation. Firstly, the composition and morphology, which influence the optical properties of material and that determine the internal volumetric energy distribution. Secondly, the above-mentioned composition and morphology are also involved in energy transport and mediate the thermomechanical response of biomaterial to pulsed laser heating and phase transformation [7].

Collagen is the most abundant animal protein and it accounts for approximately $25 \%$ of all proteins in human's body [8-10]. The abundance and structural properties of collagen have led to a wide range of uses, including leather, sutures, barrier membrane, shield, sponges, systems for tissue engineering, and haemostatics [11-19].

Poly(vinyl pyrrolidone) (PVP), because of its outstanding absorption and complexing capabilities, is very useful in pharmacy and medicine. PVP is used for the preparation of synthetic plasmas (substitute of plasma blood), in creation of hydrogels by radiation methods, and in creation of thromboresistant hydrophilic gels [20].

Collagen and poly(vinyl pyrrolidone) are well known for their interesting biological properties [21]. The abovementioned composition makes it possible to obtain new materials with strong interactions between biological and synthetic components $[22,23]$.

For the sake of the essential role played by surfaces in biomedicine, the property changes taking place on it are important in determining the suitability of biopolymers for many applications such as adhesion, protective coatings, and thin film technology.

The aim of this work is to study the interactions between $\mathrm{KrF}$ excimer laser irradiation $(\lambda=248 \mathrm{~nm})$, collagen, and collagen/PVP films (in the weight ratio of $1: 1$ ) using optical microscopy $(\mathrm{OM})$ and scanning electron microscopy (SEM).

\section{MATERIALS AND METHODS}

Collagen was obtained in our laboratory from tail tendons of young albino rats. After washing in distilled water, tendons were dissolved in $0.4 \mathrm{M}$ acetic acid solution, then spun at $8000 \mathrm{rpm}$ in a centrifuge and the soluble fraction was decanted. Poly(vinyl pyrrolidone) (360.000 molecular weight) 
was supplied by the Fluka company. The polymeric blend was prepared by mixing appropriate volumes of collagen and PVP aqueous solutions and the final weight ratio of blended polymeric substances was $1: 1$. Polymer thin films were obtained by casting solutions onto glass plates. After evaporation, the samples were dried in vacuum at room temperature. The water contents of the various films were estimated at $\sim 15 \%$ according to the method described in [24].

Laser irradiation was carried out using an excimer laser system, LPX220i (Lambda Physik) operated at $248 \mathrm{~nm}(\mathrm{KrF})$. The pulse duration was 25 nanoseconds FWHM (full width at half maximum). The laser beam was homogenized with a cylindrical lens and passed through an aperture to obtain region of uniform intensity (method described in detail in [24]). The laser irradiation was carried out in air at room temperature. Pulsed laser irradiation of collagen and its blend were performed at one hertz using various number of pulses and fluences.

The changes on film surfaces after pulsed laser irradiation were examined by means of scanning electron microscopy (SEM magnification in a case of Figures 8,11 , and 12 was $1200 \mathrm{x}$ whereas for Figures 9 and 10 was 4600 and $43000 \mathrm{x}$, resp.) and camera interfaced to a computer. The latter device allowed recording the pictures immediately after pulses. Based on some obtained images, we may consider one of the possible mechanisms, which would be involved in ablation process.

\section{RESULTS AND DISCUSSION}

Figures 3-7 show the images recorded for collagen and collagen/PVP films by means of CCD camera, immediately after $\mathrm{KrF}$ laser pulse or pulses. The fluences used for examined films ranged from $0.4 \mathrm{~J} / \mathrm{cm}^{2}$ to $1.7 \mathrm{~J} / \mathrm{cm}^{2}$. The use of single pulse of UV radiation below ablation threshold (in both cases $0.5 \mathrm{~J} / \mathrm{cm}^{2}$ ) does not cause any visible change into the surface structure of collagen as well as its blend films. Moreover, the AFM images (not shown in this paper) did not also reveal any changes in the irradiated region at a fluence of $0.4 \mathrm{~J} / \mathrm{cm}^{2}$. The first symptoms of surface modification appear after three pulses of laser light with the energy of $0.4 \mathrm{~J} / \mathrm{cm}^{2}$ each. Along with increasing number of pulses, the changes on the irradiated surfaces become more visible and at the same time we obtain a good homogeneity of fluence over the entire spot. Further increase of radiation dose (at the same energy) leads to the surface ruptures of the examined films (Figures 3 and 4) as a result of significant pressure effects generated by incident laser pulse or pulses. Beginning from the threshold value, single $\mathrm{KrF}$ laser pulses are quite sufficient to create a modified layer on the surface of a collagen film and its blend with PVP. Moreover, along with increasing pulse energy and irradiation doses, the effect of a film damage occurs at a smaller number of the incident pulses. It seems that collagen/PVP films are more fragile and slightly resistant to the influence of laser light in comparison with collagen film. Characteristic of the exposure to UV pulses biopolymer samples is a deposition of products, which were ejected from the surface outside the irradiated spot during ablation

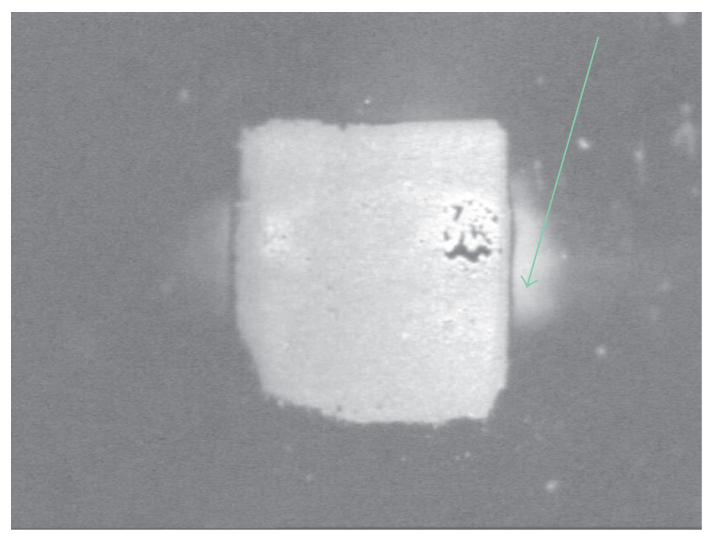

FIGURE 1: Collagen film surface after laser treatment $\left(F=0.4 \mathrm{~J} / \mathrm{cm}^{2}\right.$, 30 pulses). Green arrow indicates a material deposition; black area represents nonirradiated region of the collagen of the collagen film. On the right in the irradiated spot, the surface ruptures are seen.

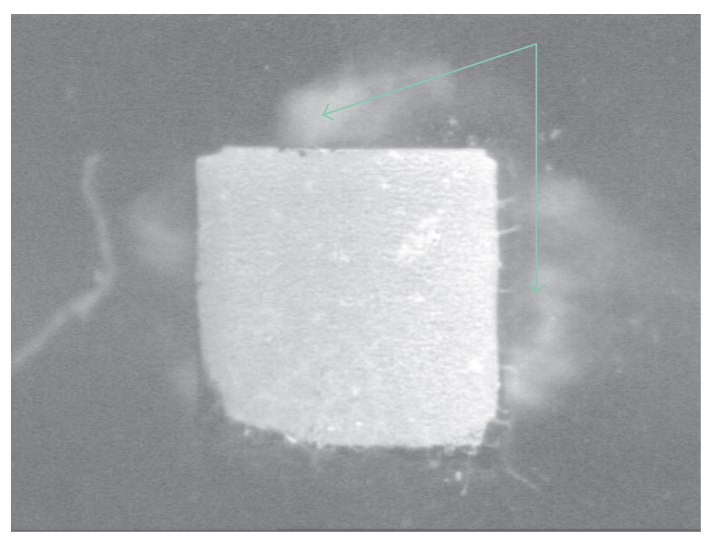

Figure 2: Collagen film surface after laser treatment $\left(F=0.7 \mathrm{~J} / \mathrm{cm}^{2}\right.$, 10 pulses). Green arrows indicate a material deposition; black area represents nonirradiated region of the collagen film.

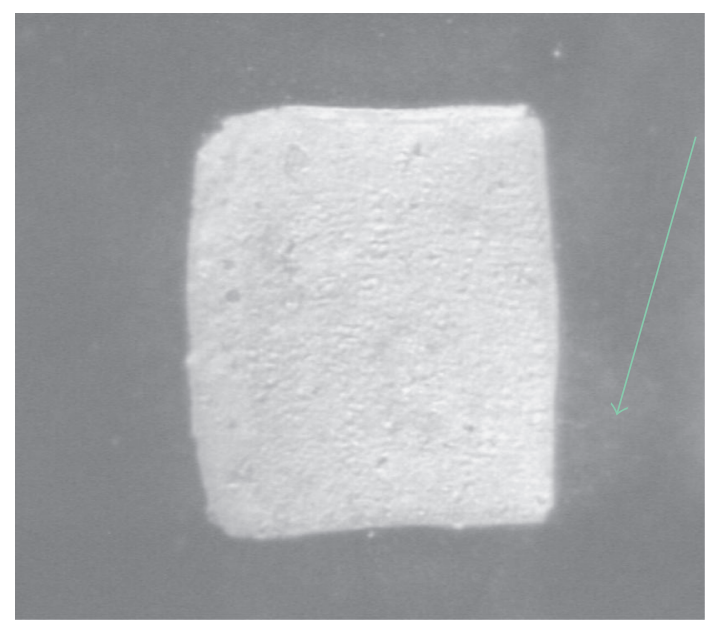

FIGURE 3: Collagen film surface after laser treatment $\left(F=1.4 \mathrm{~J} / \mathrm{cm}^{2}\right.$, 3 pulses). Green arrow indicates a material deposition; black area represents nonirradiated region of the collagen film. 


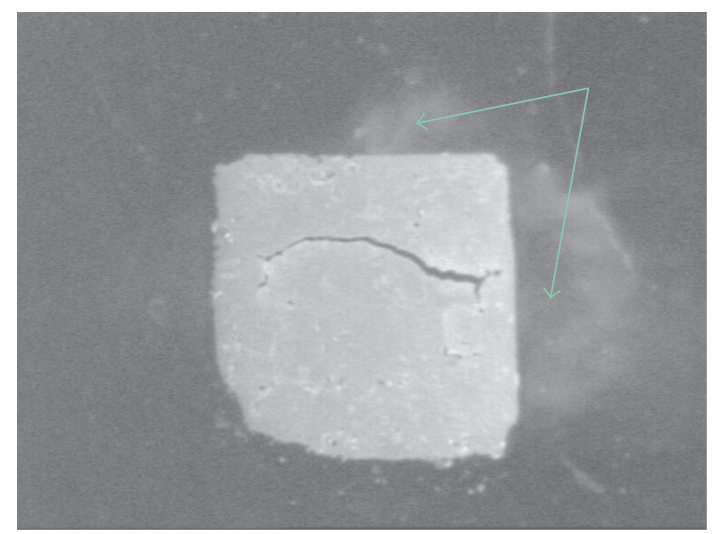

Figure 4: Collagen/PVP film surface after laser treatment $(F=$ $0.4 \mathrm{~J} / \mathrm{cm}^{2}, 30$ pulses). Green arrows indicate a material deposition; black area represents nonirradiated region of the collagen/PVP film. In the middle of the irradiated spot, the surface rupture is seen.

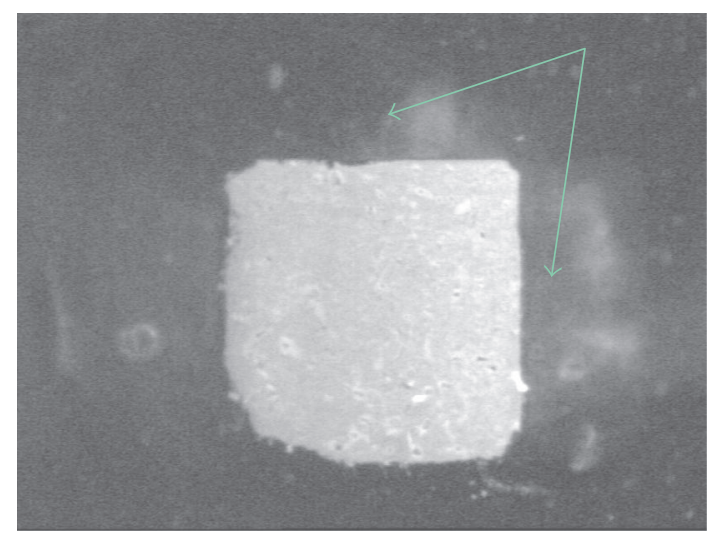

Figure 5: Collagen/PVP film surface after laser treatment $(F=$ $0.7 \mathrm{~J} / \mathrm{cm}^{2}, 15$ pulses). Green arrows indicate a material ejection; black area represents nonirradiated region of the collagen/PVP film.

process. It takes place with the first laser pulses at fluence in the range of $0.7 \mathrm{~J} / \mathrm{cm}^{2}-1.7 \mathrm{~J} / \mathrm{cm}^{2}$ and this effect becomes extraordinary if we use their increasing number (Figures 2 and 5, marked using green arrows). In a case of subthreshold values, the above-mentioned phenomenon is observed using 10 and about 15 pulses for collagen and collagen/PVP films, respectively. It should be emphasized that the use of relatively large energies $\left(1.1-1.7 \mathrm{~J} / \mathrm{cm}^{2}\right)$ causes the material ejection from the surface to occur at a higher velocity and a longer distance, therefore, an accumulation of ablation products outside the irradiated spot might be smaller (very thin layer) in comparison with the used energies of $0.4-0.7 \mathrm{~J} / \mathrm{cm}^{2}$. This effect can be seen well in the figures, which show the ruptured film surfaces (Figures 3, 3, 4, and 6, marked using green arrows). Like previously, the material ejection under the influence of incident laser pulses seems to be slightly more efficient in a case of collagen films (Figures 2 and 5).

For PVP films, pulses at a fluence of $0.4-0.7 \mathrm{~J} / \mathrm{cm}^{2}$ do not cause any surface modification, even if we supply them with

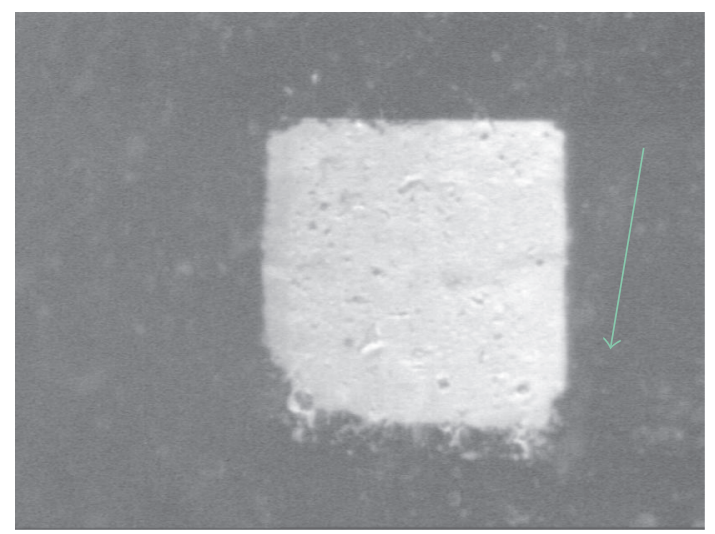

FIGURE 6: Collagen/PVP film surface after laser treatment $(F=$ $1.4 \mathrm{~J} / \mathrm{cm}^{2}, 3$ pulses). Green arrow indicates a material ejection; black area represents nonirradiated region of the collagen/PVP film.

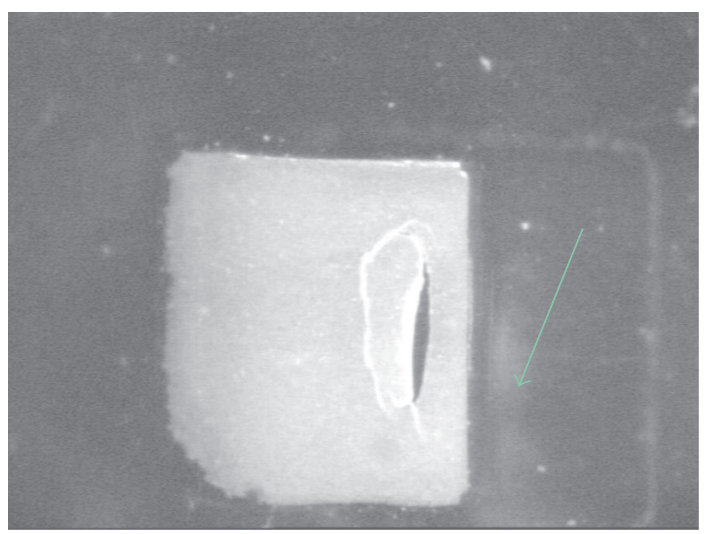

Figure 7: PVP film surface after laser treatment $\left(F=1.7 \mathrm{~J} / \mathrm{cm}^{2}, 60\right.$ pulses). Green arrow indicates a material ejection; black area represents nonirradiated region of PVP film. On the right in the irradiated spot, the surface ruptures are seen.

large number of pulses. The first evidence of surface alteration appears after 5,2 , and 1 pulses with the energies of 1.1; $1.4 ; 1.7 \mathrm{~J} / \mathrm{cm}^{2}$, respectively. The film damages occur at the enormous number of pulses (70-60) compared to collagen and collagen/PVP films. Similarly, the quantity of material ejection from the irradiated surface is not large in relation to the irradiated collagen and its blend. It can be seen using 3070 pulses of UV light (Figure 7, marked using green arrow).

Based on the obtained results, we can conclude that PVP is a more resistant material to $\mathrm{KrF}$ laser irradiation than collagen and collagen/PVP. The evidence is the threshold value, which far exceeds values obtained for collagen and collagen/PVP films. Moreover, the surface modification connected with the materials ejection from the ablated spot requires significantly larger numbers of pulses. Blending it with $50 \%$ of PVP causes its ablation resistance to increase in a small extent only. The threshold values for collagen and collagen/PVP films are of the same order. It is in a good agreement with our previous results [23] that collagen 


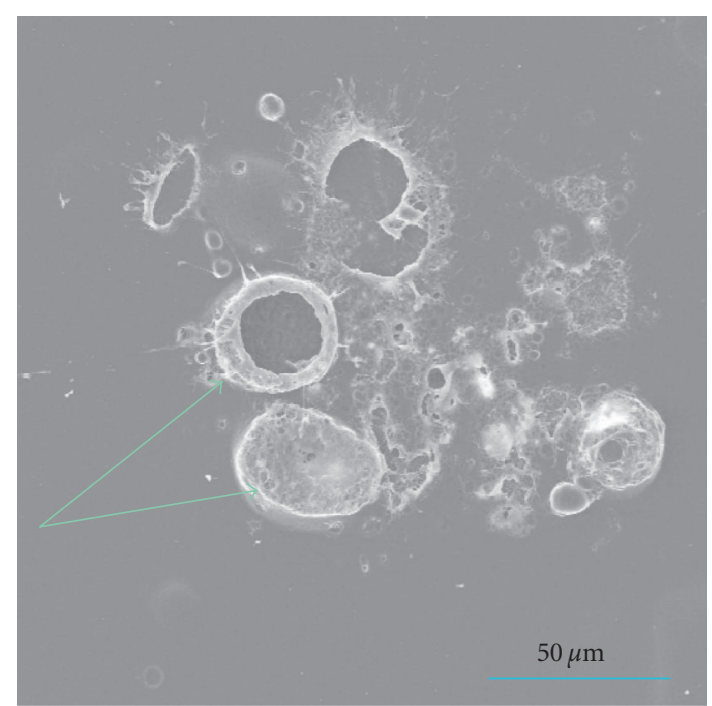

FIGURE 8: SEM image of collagen/PVP film surface after laser irradiation $\left(F=0.4 \mathrm{~J} / \mathrm{cm}^{2}, 2\right.$ pulses, scale bar is $\left.50 \mu \mathrm{m}\right)$. Green arrows show spots after bubbles formation and their subsequent collapse.

predominates on the top surface layer of the examined blend (XPS results, not published yet). All results including energies and the number of pulses used with the short description of effects in Table 1 were collected.

Figures 8-12 show the SEM images recorded for collagen and collagen/PVP film surfaces. Film surfaces were exposed to single pulses of $\mathrm{KrF}$ laser radiation with increasing fluence. The range of supplied energies was $0.4-5.0 \mathrm{~J} / \mathrm{cm}^{2}$. In a case of fluence of $0.4 \mathrm{~J} / \mathrm{cm}^{2}, 3$ and 2 pulses were used.

The SEM images of the nonirradiated surface of the examined films have no irregularities and no obvious damages (outside the irradiated spot). The SEM images of biopolymers films after laser treatment are shown in Figures 8-12. UV irradiation of collagen and its blend with a fluence of $0.4 \mathrm{~J} / \mathrm{cm}^{2}$ using 3 and 2 pulses caused considerable disturbance in the surface structure which becomes very rough and includes the characteristic areas resulting from the bubbles formation and their subsequent collapse (marked using green arrows). This effect can be easily seen in Figure 8 .

The laser irradiation of the used specimens caused an expansion of materials above their initial surface with wellpronounced symptoms of its melting. SEM images and profilometry (results not shown here) of the ablated surface display an important swelling $(\sim 5-7 \mu \mathrm{m})$ and a macroscopic foam structure indicative of the laser induced expansion of the excited material. This process is due to the explosive ablation, also called explosive boiling of the irradiated material, and produces because of relatively large absorption depth $(20 \mu \mathrm{m})$ and because of the presence of a large amount of water in the material which upon laser heating generates a high concentration of bubbles within biopolymer under the action of a transient tensile wave. Further bubble colliding and bursting lead to the formation of a three-dimensional polymer "microfoam" structure with a good interconnection

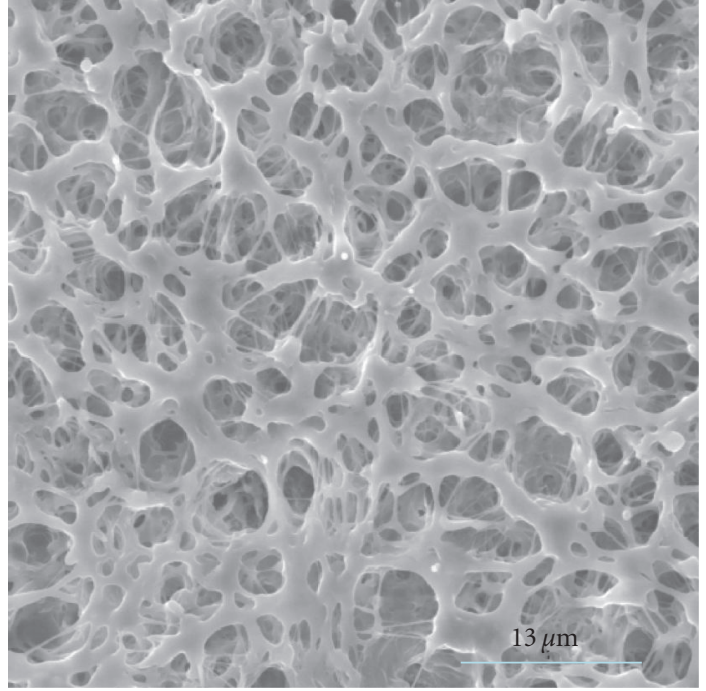

FIGURE 9: SEM image of collagen film surface after laser irradiation $\left(F=1.7 \mathrm{~J} / \mathrm{cm}^{2}, 1\right.$ pulse, scale bar is $\left.13 \mu \mathrm{m}\right)$.

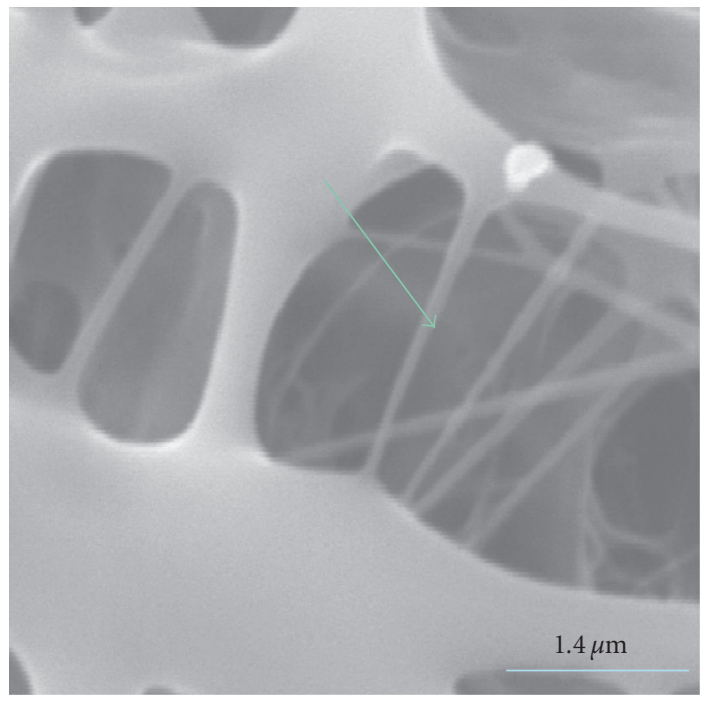

FIGURE 10: SEM image of collagen film surface after laser irradiation, $\left(F=3 \mathrm{~J} / \mathrm{cm}^{2}, 1\right.$ pulse, scale bar is $\left.1.4 \mu \mathrm{m}\right)$. Green arrow shows ultra-thin fibers with nanometric diameters.

between pores (Figure 9). The mechanism of the foaming surface is not a classical ablation process leading to a clean etching. It clearly proceeds by a dense bubble nucleation followed by a fast expansion because of strong pressure effects [24-26]. Using the physical constants for our collagen film, we estimated the temperature rise on the irradiated surface. It was surprisingly low and amounted to $141^{\circ} \mathrm{C}$ at $1.0 \mathrm{~J} / \mathrm{cm}^{2}$ and $83^{\circ} \mathrm{C}$ at $0.5 \mathrm{~J} / \mathrm{cm}^{2}$ (the threshold fluence). These temperatures are very moderate and the experimental fluence threshold of foaming is much below the normal critical energy density for water evaporation. This is a strong evidence 


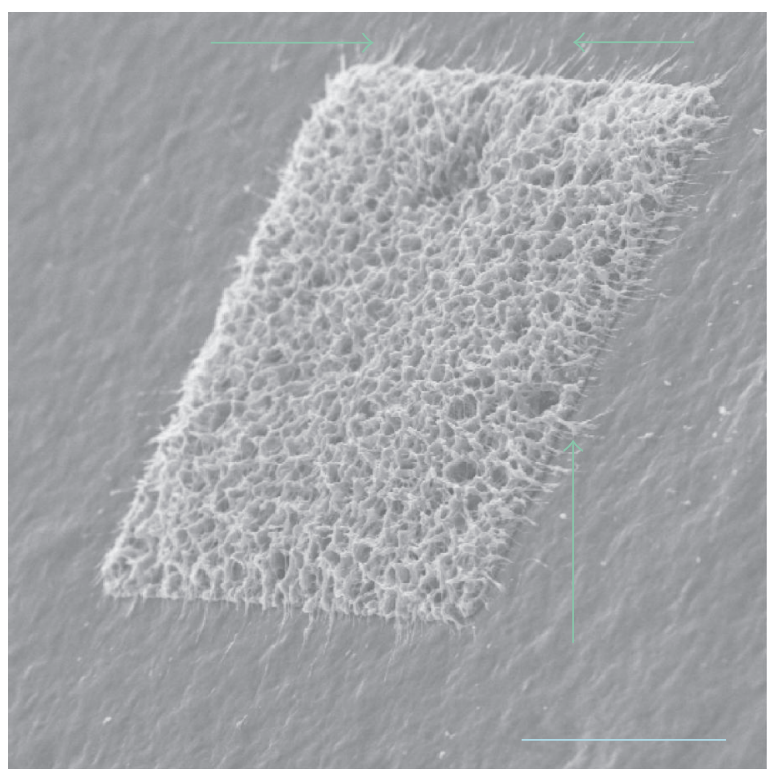

FIGURE 11: SEM image of collagen film surface after laser irradiation, $\left(F=1.7 \mathrm{~J} / \mathrm{cm}^{2}, 1\right.$ pulse, scale bar is $\left.50 \mu \mathrm{m}\right)$. Green arrows show characteristic filaments formed due to a strong gradient of fluence at the edges.

that the abnormal conditions of transient acoustic pressure must be taken into consideration [24]. More detailed information concerning the mechanism of the ablation process has been presented in our recent papers [24-26].

In Figure 10 (marked using green arrow) details of the inside of the "microfoam" structure for collagen film as a result of coalescence, formation of interfacial walls with subsequent rupture leading to ultra-thin fibers with nanometric diameters were shown. In this case, the diameter of the obtained nanofibers was about $0.1 \mu \mathrm{m}$. Similar structures were also observed for collagen/PVP films. It is also noteworthy that along with increasing energies because of good homogeneity of fluence over the entire spot and a strong gradient of fluence at the edges, the melt expulsion and characteristic filaments [27] outside the irradiated place were observed (Figures 11 and 12 marked using green arrows). It should be noticed that filaments are created at fluences larger than $0.7 \mathrm{~J} / \mathrm{cm}^{2}$. It concerns both collagen and collagen/PVP films. Moreover, the quantity of melt expulsion at the same energy $\left(1.7 \mathrm{~J} / \mathrm{cm}^{2}\right)$ and single pulse of laser light is greater in a case of collagen film. "Microfoam" structures obtained for both materials seem to be distinct which can be seen in Figures 11 and 12. For collagen/PVP film, the bubbles nucleation induced by tensile part of the photoacoustic effect occurs likely with a smaller yield, therefore, these surfaces possess smaller amount of pores. It is also connected with the quantity of materials ejected during the ablation process, which for the collagen film surface, occurs more efficiently. Thus, it seems that in the case of collagen/PVP blend, the influence of the synthetic polymer causes a small increase of resistance to the effects generated by UV laser pulse and the morphology of the irradiated region. All results including energies and

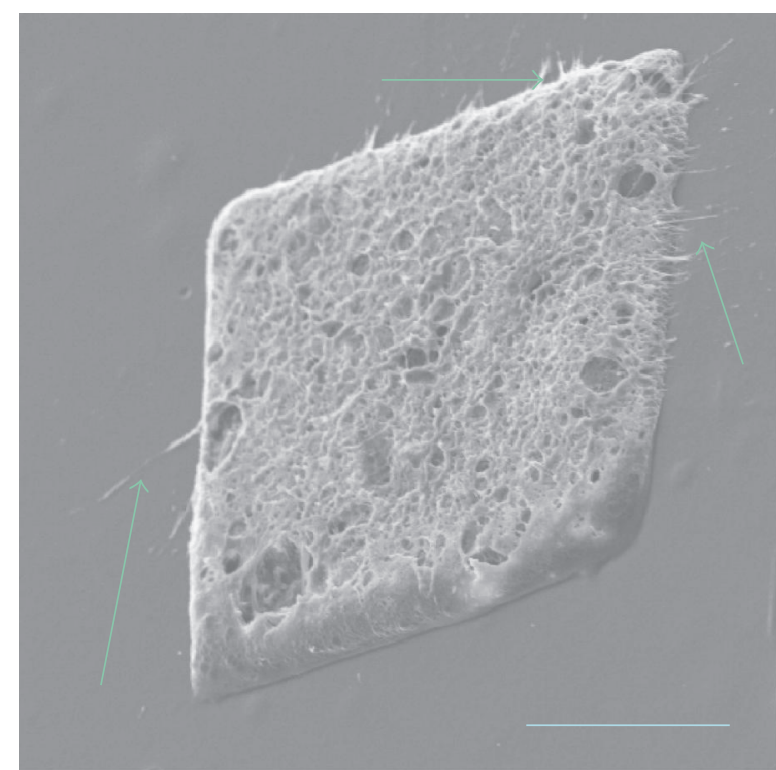

FIGURE 12: SEM image of collagen/PVP film surface after laser irradiation $\left(F=1.7 \mathrm{~J} / \mathrm{cm}^{2}, 1\right.$ pulse, scale bar is $\left.50 \mu \mathrm{m}\right)$. Green arrows show filaments at the edges of irradiated spot.

number of pulses used with the short description of effects in Table 1 were collected.

Similarities with the present surface foam structures can be found in synthetic polymer ablation [27-29].

\section{CONCLUSION}

$\mathrm{KrF}$ laser treatment of collagen and collagen/PVP films caused significant damages on their surfaces leading to the formation of "microfoam" structures as a result of an explosive thermal mechanism assisted by the negative part of laser induced photoacoustic pressure. In the above-mentioned mechanism, we considered a purely thermal pathway although the eventuality of reactions of electronic excited states (photochemical pathway) cannot be ruled out. It is due to the photon energy generated by $\mathrm{KrF}$ excimer laser $(5.0 \mathrm{eV})$, which is sufficient to direct the breaking of $\mathrm{C}-\mathrm{C}$ bonds in the main chain of collagen molecule or to cause a partial decomposition of five-membered ring of proline [30]. Both collagen and collagen/PVP films behave very similarly. Based on obtained results, we can affirm that the addition of PVP to collagen causes its resistance to increase the ablation process in a small extent only. It seems that surface foaming by laser ablation is a promising approach because it is a "green process," which does not use or reject any chemical reagent potentially contaminating for the environment. We expect that the laser induced "microfoam" structures can find some new applications in the future.

\section{ACKNOWLEDGMENTS}

Financial support from the Marie Curie Program (HPMTCT-2000-001430), POLONIUM Program, and from the 
TABLE 1: Experimental conditions and the effects of KrF laser irradiation on the studied film surfaces.

\begin{tabular}{ccc}
\hline & Techniques: CCD camera and SEM \\
\hline $\mathrm{F}\left(\mathrm{J} / \mathrm{cm}^{2}\right)$ & Pulse number & Sample: collagen film \\
\hline 0.4 & 30 & Description of effects \\
0.7 & 10 & Material deposition (Figures 3-3) and surface ruptures (Figure 3) [CCD] \\
1.4 & 3 & \\
1.7 & 1 & Surface foaming (Figures 9-11), filament (Figure 11), and ultra-thin fibres \\
3 & 1 & formation (Figure 10) [SEM] \\
\hline 0.4 & 30 & Sample: collagen/PVP blend \\
0.7 & 15 & Material deposition (Figures 4-6) and surface ruptures (Figure 4) [CCD] \\
1.4 & 3 & \\
0.4 & 2 & Spots after bubbles formation and their subsequent collapse (Figure 8), \\
1.7 & 1 & surface foaming and filament formation (Figure 12) [SEM] \\
\hline 1.7 & 60 & Sample: PVP film \\
\hline
\end{tabular}

Ministry of Science and Information Society Technologies (MNII, Poland) Grant number 3T08E 3829 is gratefully acknowledged.

\section{REFERENCES}

[1] J. M. Krauss, C. A. Puliafito, and R. F. Steinert, "Laser interactions with the cornea," Survey of Ophthalmology, vol. 31, no. 1, pp. 37-53, 1986.

[2] C. A. Puliafito, R. F. Steinert, T. F. Deutsch, F. Hillenkamp, E. J. Dehm, and C. M. Adler, "Excimer laser ablation of the cornea and lens. Experimental studies," Ophthalmology, vol. 92, no. 6, pp. 741-748, 1985.

[3] J. Marshall, S. Trokel, S. Rothery, and H. Schubert, "An ultrastructural study of corneal incisions induced by an excimer laser at 193 nm," Ophthalmology, vol. 92, no. 6, pp. 749-758, 1985.

[4] M. S. Kitai, V. L. Popkov, V. A. Semchishen, and A. A. Kharizov, "The physics of UV laser cornea ablation," IEEE Journal of Quantum Electronics, vol. 27, no. 2, pp. 302-307, 1991.

[5] Proceedings of the 5th International Conference on Laser Ablation (COLA '99), J. S. Horwitz, H. U. Krebs, K. Murakami, and M. Stuke, Eds., Appl. Phys. A69 Suppl., Gottingen, Germany, July 1999.

[6] S. L. Trockel, R. Srinivasan, and B. Braren, "Excimer laser surgery of the cornea," American Journal of Ophthalmology, vol. 96, p. 710, 1983.

[7] A. Vogel and V. Venugopalan, "Mechanisms of pulsed laser ablation of biological tissues," Chemical Reviews, vol. 103, no. 2, pp. 577-644, 2003.

[8] B. Alberts, D. Bray, J. Lewis, M. Raff, K. Roberts, and J. D. Watson, Molecular Biology of the Cell, Garland, New York, NY, USA, 3rd edition, 1994.

[9] D. A. D. Parry and A. S. Craig, Ultrastructure of the Connective Tissue Matrix, edited by A. Ruggeri and P. M. Motta, Martinus Nijhoff, Boston, Mass, USA, 1984.
[10] M. Van der Rest and R. Garrone, "Collagen family of proteins," FASEB Journal, vol. 5, no. 13, pp. 2814-2823, 1991.

[11] T. Miyata, T. Taira, and Y. Noishiki, "Collagen engineering for biomaterial use," Clinical Materials, vol. 9, no. 3-4, pp. 139 148, 1992.

[12] A. L. Rubin, K. H. Stenzel, T. Miyata, M. J. White, and M. Dune, "Collagen as a vehicle for drug delivery: preliminary report," Journal of Clinical Pharmacology, vol. 13, no. 8-9, pp. 309-312, 1973.

[13] S. E. Bloomfield, T. Miyata, M. W. Dunn, N. Bueser, K. H. Stenzel, and A. L. Rubin, "Soluble gentamicin ophthalmic inserts as a drug delivery system," Archives of Ophthalmology, vol. 96, pp. 885-887, 1978.

[14] C. I. Wedge and D. S. Rootman, "Collagen shields: efficacy, safety and comfort in the treatment of human traumatic corneal abrasion and effect on vision in healthy eyes," Canadian Journal of Ophthalmology, vol. 27, no. 6, pp. 295-298, 1992.

[15] G. J. Angella, M. B. Sherwood, L. Balasubramanian, et al., "Enhanced short-term plasmid transfection of filtration surgery tissues," Investigative Ophthalmology and Visual Science, vol. 41, no. 13, pp. 4158-4162, 2000.

[16] M. Kuwano, Y. Horibe, and Y. Kawashima, "Effect of collagen cross-linking in collagen corneal shields on ocular drug delivery," Journal of Ocular Pharmacology and Therapeutics, vol. 13, no. 1, pp. 31-40, 1997.

[17] J. C. Geesin, L. J. Brown, Z. Liu, and R. A. Berg, "Development of a skin model based on insoluble fibrillar collagen," Journal of Biomedical Materials Research, vol. 33, no. 1, pp. 1-8, 1996.

[18] F. A. Auger, M. Rouabhia, F. Goulet, F. Berthod, V. Moulin, and L. Germain, "Tissue-engineered human skin substitutes developed from collagen-populated hydrated gels: clinical and fundamental applications," Medical and Biological Engineering and Computing, vol. 36, no. 6, pp. 801-812, 1998.

[19] S. T. Boyce, "Skin substitutes from cultured cells and collagenGAG polymers," Medical and Biological Engineering and Computing, vol. 36, no. 6, pp. 791-800, 1998. 
[20] J. Rosiak, K. Burczak, J. Olejniczak, and W. Pekala, "Polymer materials for biomedical use obtained by radiation methods. III. Radiation cross-linking of acryloamide and Nvinylpyrrolidone," Polimery w Medycynie, vol. 19, p. 3, 1989.

[21] C. R. Cervantes-Sánchez, E. Olaya, M. Testas, et al., "CollagenPVP, a collagen synthesis modulator, decreases intraperitoneal adhesions," Journal of Surgical Research, vol. 110, no. 1, pp. 207-210, 2003.

[22] A. Sionkowska, "Interaction of collagen and poly(vinyl pyrrolidone) in blends," European Polymer Journal, vol. 39, no. 11, pp. 2135-2140, 2003.

[23] A. Sionkowska, H. Kaczmarek, M. Wisniewski, J. Kowalonek, and J. Skopinska, "Surface characteristics of UV-irradiated collagen/PVP blended films," Surface Science, vol. 566-568, no. 13, part 1, pp. 608-612, 2004.

[24] S. Lazare, V. Tokarev, A. Sionkowska, and M. Wisniewski, "Surface foaming of collagen, chitosan and other biopolymer films by $\mathrm{KrF}$ excimer laser ablation in the photomechanical regime," Applied Physics A: Materials Science and Processing, vol. 81, no. 3, pp. 465-470, 2005.

[25] S. Lazare, V. Tokarev, A. Sionkowska, and M. Wisniewski, "Negative pressure model for surface foaming of collagen and other biopolymer films by KrF laser ablation," to appear in Journal of Physics: Conference Series.

[26] S. Lazare, A. Sionkowska, M. Wisniewski, V. Tokarev, and M. Castillejo, "Single laser pulse induced cavitation, bubbling and foaming on biopolymer films," to appear in Journal of Laser Micro/Nanoengineering.

[27] F. Weisbuch, V. N. Tokarev, S. Lazare, C. Belin, and J. L. Bruneel, "Millimeter-long nanofibers of PMMA spun at super-high speed by ablation with a single pulse of a KrF excimer laser," Applied Physics A: Materials Science and Processing, vol. 75, no. 6, pp. 677-680, 2002.

[28] H. Hiraoka, S. Lazare, T. J. Chuang, C. T. Rettner, and H. E. Hunziker, "Laser photoetching of doped poly(tetrafluoroethylene), substituted-PTFE, and polyimide films," Microelectronic Engineering, vol. 13, no. 1-4, pp. 429-432, 1991.

[29] P. E. Dyer, D. M. Karnakis, G. A. Oldershaw, and G. C. Roberts, "TEA $\mathrm{CO}_{2}$ laser ablation of Upilex polyimide," Journal of Physics D: Applied Physics, vol. 29, no. 10, pp. 2554-2563, 1996.

[30] M. Wisniewski, A. Sionkowska, H. Kaczmarek, S. Lazare, and V. Tokarev, "The influence of UV laser radiation on the thin collagen films: mechanism and spectroscopic studies," to appear in Polimery (Polish Journal). 


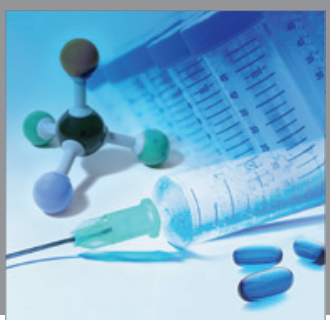

International Journal of

Medicinal Chemistry

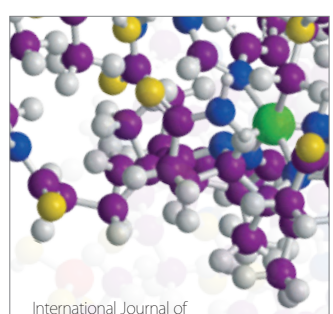

Carbohydrate Chemistry

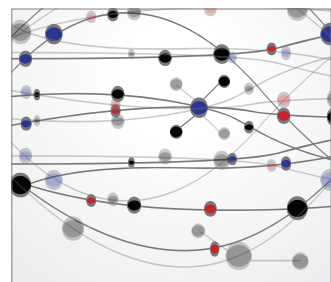

The Scientific World Journal
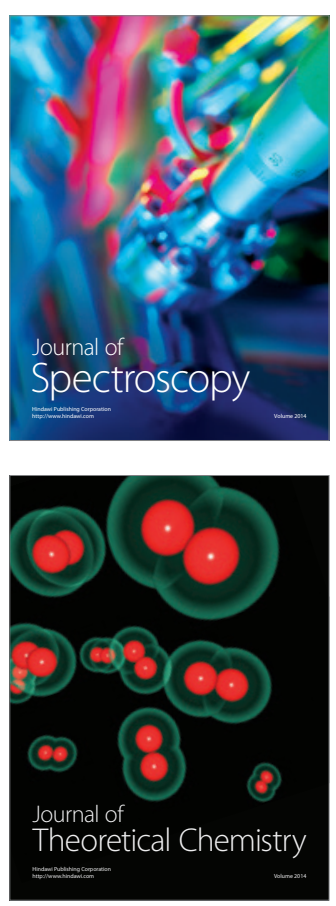
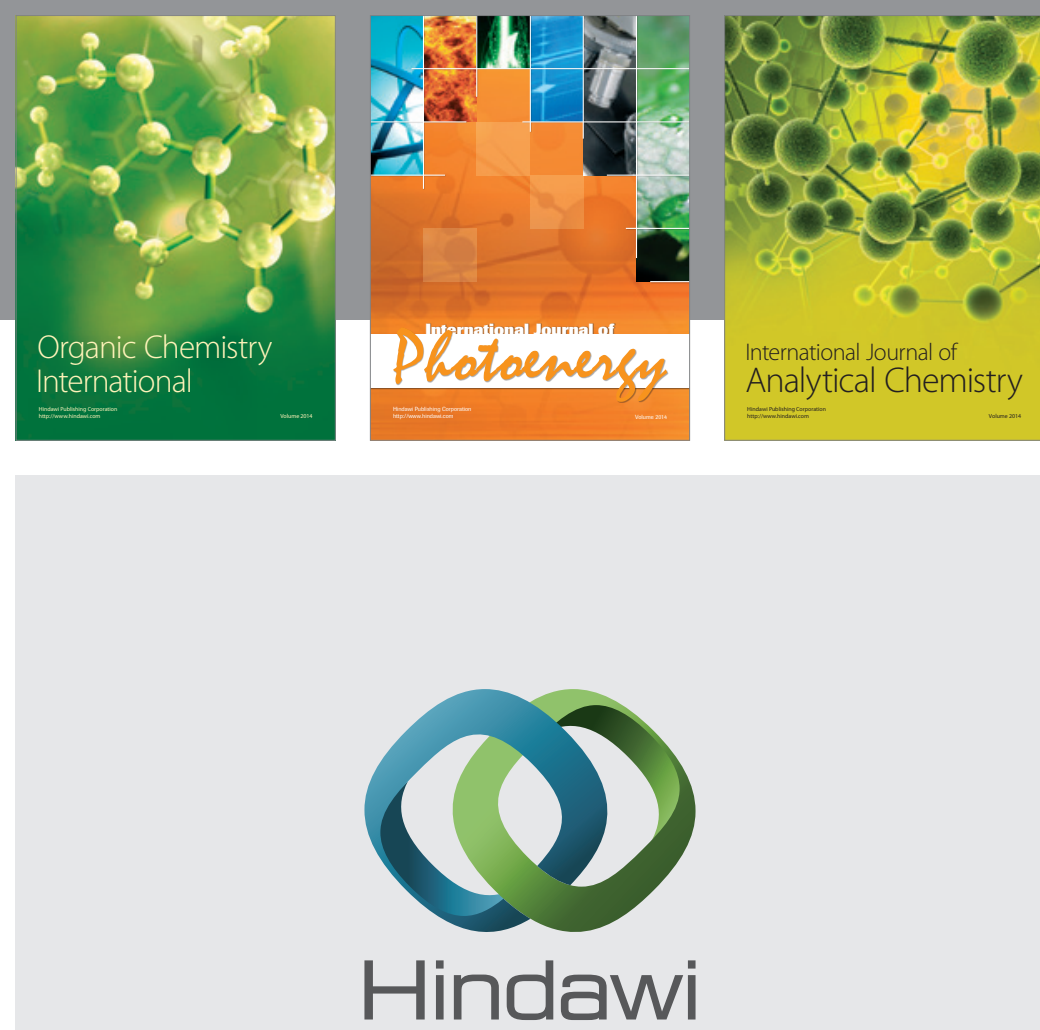

Submit your manuscripts at

http://www.hindawi.com
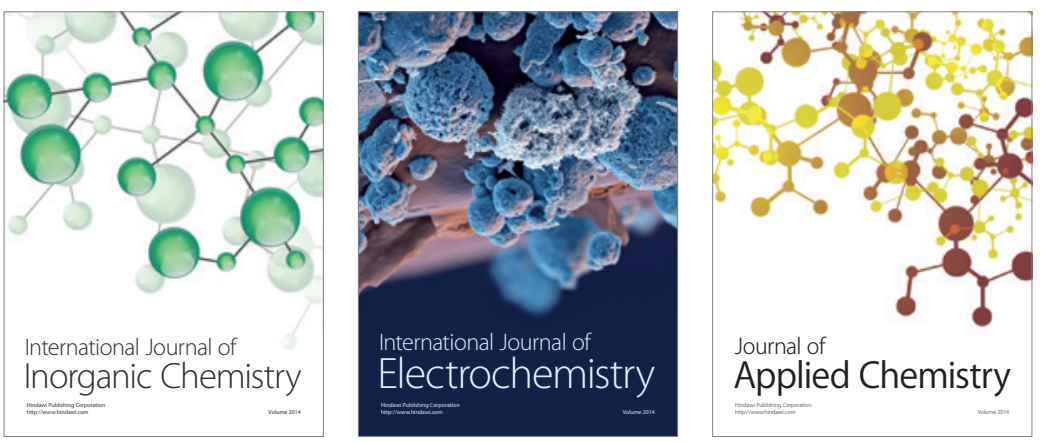

Journal of

Applied Chemistry
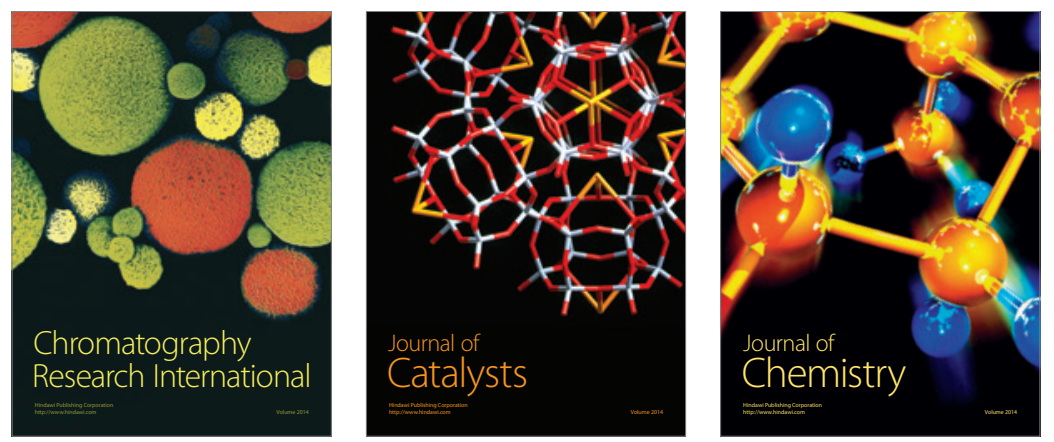
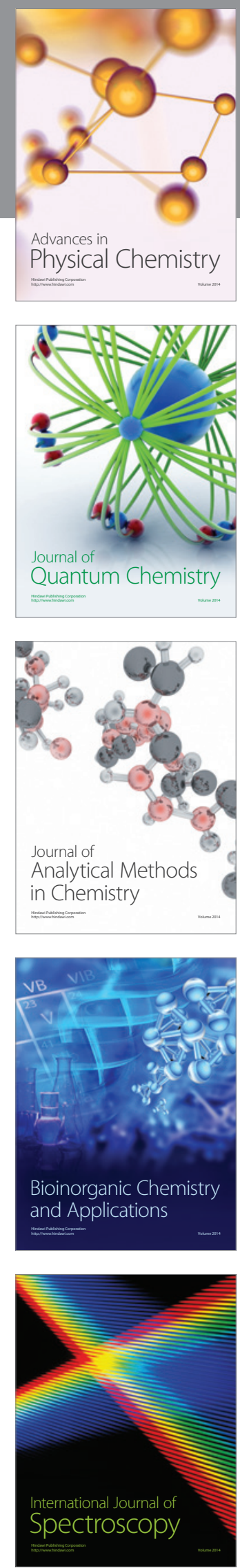